\title{
1-Jahres-Daten zu Upadacitinib
}

Unter kontinuierlicher Therapie mit dem oralen, selektiven und reversiblen Januskinase (JAK)-Inhibitor Upadacitinib waren $29 \%$ der Patienten mit Psoriasis-Arthritis (PsA) und vorangegangenem BiologikaVersagen auch nach einem Jahr in einem Zustand minimaler Krankheitsaktivität (MDA) - so das beim virtuellen EULAR-Kongress 2021 vorgestellte Ergebnis der SELECT-PsA 2-Studie.

Diese Daten sind vor dem Hintergrund, dass Upadacitinib vor Kurzem in der EU die Zulassung für die Indikation Psoriasis-Arthritis erhalten hat, wichtig. In der multizentrischen, doppelblinden, placebokontrollierten Phase III-Studie SELECT-PsA 2 waren PsA-Patienten mit nicht ausreichendem Ansprechen auf $\geq 1$ Biologikum beziehungsweise mit

\section{Die aktuelle Auswertung}

Die jetzige Auswertung [1] zu Woche 56 zeigt, dass $60 \%$ der 211 PsA-Patienten, die kontinuierlich mit Upadacitinib behandelt worden waren, weiterhin ein ACR20-Ansprechen aufwiesen. Ein ACR50-Ansprechen hatten $41 \%$ erreicht, ein ACR70-Ansprechen $24 \%$. Eine MDA wiesen nach einem Jahr $29 \%$ der Patienten auf. insgesamt war das Ansprechen unter $15 \mathrm{mg}$ mit dem unter $30 \mathrm{mg}$ vergleichbar und hatte sich bis Woche 56 dem unter $30 \mathrm{mg}$ angeglichen.

Auch für die anderen Krankheitsdomänen fand sich eine hohe Therapieeffizienz. Der Anteil der Patienten, bei denen es unter Upadacitinib zu einem Abklingen von Enthesitis, Daktylitis bzw. einer Abheilung der Hauterscheinungen gekommen war, blieb bis zum Ende der einjährigen Behandlungsphase konstant. Es fanden sich keine neuen Sicherheitssignale im Vergleich zur Auswertung in Woche 24. Das Sicherheitsprofil entsprach dem in früheren Studien und denen bei der rheumatoiden Arthritis.

\section{Literatur \\ 1. Mease PJ et al (2021) Upadacitinib In Patients With Psoriatic Arthritisrefractory To Biologic Disease-Modifyingantirheumatic Drugs: 56-Week Data From The Phase 3Select-Psa 2 Study. EULAR 2021, POS0196}

Hinweis des Verlags. Der Verlag bleibt in Hinblick auf geografische Zuordnungen und Gebietsbezeichnungen in veröffentlichten Karten und Institutsadressen neutral.

rheuma plus $2021 \cdot 20: 150$ https://doi.org/10.1007/s12688-02100448-5

(c) Springer-Verlag GmbH Austria, ein Teil von Springer Nature 2021

Hier steht eine Anzeige. 